\title{
Answer to the comment by Yoshisada Shibata on "Hiroshima survivors exposed to very low doses of A-bomb primary radiation showed a high risk for cancers"
}

\author{
Masaru Miyao - Tomoyuki Watanabe · \\ Ryumon Honda · Yuichi Yamada
}

Received: 19 November 2008 / Accepted: 2 December 2008/Published online: 29 January 2009

(C) The Japanese Society for Hygiene 2009

Yoshisada Shibata raises four issues with regard to our paper "Hiroshima survivors exposed to very low doses of A-bomb primary radiation showed a high risk for cancers" [1], which we would like to discuss.

(1) Use of standardized mortality ratio (SMR) for comparisons

The SMR is an indirect method based on the age structure of the target population. It is a ratio of the observed $(\mathrm{O})$ to the expected $(\mathrm{E})$ number of deaths, where $\mathrm{E}$ is the number of individuals of the target population multiplied by the age- and sex-specific mortality rates of the standard population. In his commentary, Shibata wrote that "The authors should have compared the mortality directly between the target population and a common standard population,...". We are unclear what is meant by this statement. If we were to compare age-period specific mortality rates for each age group (seven classes) and calendar year (five periods), we would need to analyze 35fold (seven age-classes times five periods) more data than that analyzed in the reported study. The result of such a mega-analysis would be to diffuse the focus of our study, with the reader ultimately having great difficulty

M. Miyao ( $\square)$

Information Technology Center, Nagoya University,

Furo-cho, Chikusa-ku, Nagoya 464-8601, Japan

e-mail: mmiyao@med.nagoya-u.ac.jp

T. Watanabe

Department of Health and Nutrition,

Faculty of Psychological and Physical Science,

Aichi Gakuin University, Nisshin, Japan

R. Honda · Y. Yamada

Department of Social and Environmental Medicine (Hygiene),

Kanazawa Medical University, Ishikawa, Japan understanding the results. A comparison using SMR may not have been the best approach, but we could not have achieved the aim of our study if we did not use summary rates.

Shibata said that "the effects of the age distribution in the target population on the power of the hypothesis test are considerably large". The test of significance was performed appropriately in our paper. Therefore, his comment is unrelated to the validity of the analysis in our study.

If, as pointed out by Shibata, "a relatively large portion of LSS subjects have been living in areas other than Hiroshima or Okayama prefecture," the results may have been the same-even if other prefectures were used as the standard population. Therefore, we also believe that if information were to be provided on the percentage of the LSS group living in areas other than Hiroshima and Okayama prefectures, this would be valuable data for discussion and future research.

\section{(2) Mortality study and incidence study}

A follow-up on the deaths of people who migrated out of Hiroshima or Nagasaki prefecture has been conducted in LSS. Since LSS is a large-scale cohort study, it has several advantages over studies using SMR, which can not consider the variation of death and incidence data by population migration. The unexposed subjects that Shibata puts forward are the so-called NIC (not in the city at the time of bombing). We wrote in our paper that all LSS reports after report 8 have estimated the risk of radiation exposure among A-bomb survivors using regression analyses. These analyses, however, did not show the results for A-bomb survivors in comparison with an unexposed group (NIC) [2]. The LSS reports use the term "unexposed group", but this category of survivors was actually exposed to low-dose primary radiation. The LSS has not published a report in 
which the LSS group was compared with NIC for all causes of death only since the report by Cologne and Preston [3]. Since then, the LSS has not released the results of studies using the NIC, primarily because the NIC was found to be significantly different from the exposed group (LSS group) with respect to socio-economic status and life style. If LSS had followed a genuinely appropriate unexposed group as the cohort instead of NIC, and the true exposure risk was analyzed by dosage and distance, our study may not have been necessary.

Shibata wrote: "note that the quality of mortality data is essentially the same as that of the vital statistics they used". However, because the death data were based on death certificates recorded by physicians, he can not insist that the quality of the data was "essentially the same" unless he has some way to rule out the possibility of measure bias by physicians, a point we indicated in our Discussion.

\section{(3) Defects of the LSS study}

Shibata said that a genuine attempt was made in performing the risk analysis in LSS without using an unexposed group. He also described the background of the LSS was to estimate the "risk of the unexposed group" by extrapolation of the results for the exposed group using a "sophisticated" statistical analysis, Poisson regression analysis-without using a genuine unexposed group.

Because the NIC (original control group) significantly differed from the exposed group in terms of socio-economic status and life style, the former could not be used as control group in LSS. The results of the analysis adjusted by these covariates, however, should be published if data on individuals were collected in LSS. To extrapolate the results of a regression curve goes against a basic principle of statistics. Shibata said that "it is not unusual from the scientific viewpoint to extrapolate the results to $0 \mathrm{~Sv}^{\text {", }}$, but, in fact, it is unusual.

Even if we were to admit that it could be acceptable to estimate the risk of $0 \mathrm{~Sv}$ (unexposed control group) by extrapolating from the exposed group only, under which conditions would this be acceptable? Would it not be when we knew the accurate radiation dose and its risk in a group exposed to a very low radiation dose that was close to $0 \mathrm{~Sv}$ ? However, the effects of residual radiation, etc., were ignored in LSS, which is a study based on dose estimation by DS86 of the primary radiation exposure. The risk for $0 \mathrm{~Sv}$ (unexposed control group) is overestimated as the underestimated risk estimation of the exposed group becomes higher.

In our study, the cancer risk of Hiroshima survivors exposed to a very low dose of A-bomb primary radiation (in fact, the group certified as "very low dose" by DS86 had higher exposure), seems to be much higher than that of a genuine unexposed control group. Therefore, the possibility is supported that the "unexposed subjects" in LSS were, in fact, exposure victims with a considerably higher risk than the general population.

\section{(4) Post hoc argument}

This comment reminds us of the dispute between "falsificationism," advanced by Popper [4], and classical "inductivism" in the philosophy of science. Shibata's position may be closer to the "falsificationist" position of Popper, but this philosophical dispute is by no means unsettled.

The physical measurement data on residual radiation in the Koi and Takasu areas that Shibata mentions are not those of radioactivity that reached the ground when the survivors were exposed-rather, they were those that lasted there despite later weathering. The survivors were exposed to radioactive substances carried by radioactive rain as well as radioactive fine particles hanging in the air. These fine particles were carried away by wind, while substances on the ground were partially washed away by rain due to the firestorm and decreased further by being washed away in later rains from typhoons and other storms.

Radioactive particles are easily taken into the body and have large effects on people exposed to them, but this was not measured in the Hiroshima atomic bombing. If physical measurements can not be obtained, the only possibility is to estimate the effects from the results of second-best methods, such as in our study. If those results can be demonstrated to be true after being exposed to falsification from the results of other studies, then this approach was correct in terms of the advancement of learning.

\section{(5) Central point of our paper}

The major point of our paper is that Hiroshima survivors exposed to very low doses of A-bomb primary radiation (DS86) showed a higher risk for cancers than did genuine unexposed control groups (Hiroshima and Okayama residents). Shibata had no comment on this point, leaving us to wonder what he thinks of the major theme of our study.

\section{References}

1. Watanabe T, Miyao M, Honda R, Yamada Y. Hiroshima survivors exposed to very low doses of A-bomb primary radiation showed a high risk for cancers. Environ Health Prev Med. 2008;13:264-70.

2. Pierce DA, Shimizu Y, Preston DL, Vaeth M, Mabuchi K. Studies of the mortality of atomic bomb survivors. Report 12, Part I. Cancer: 1950-1990. Radiat Res. 1996;146:1-27.

3. Cologne JB, Preston DL. Impact of comparison group on cohort dose response regression: an example using risk estimation in atomic-bomb survivors. Health Phys. 2001;80:491-6.

4. Popper K. The logic of scientific discovery. New York: Basic Books; 1959. 